\title{
Physically based criterion for prediction of instability under stretch- bending of sheet metal
}

\author{
E.S. Perdahcıoğlu ${ }^{1, a^{*}}$, B. Hou ${ }^{2, b}$ and A.H. van den Boogaard ${ }^{1, c}$ \\ ${ }^{1}$ University of Twente, P.O. Box, 7500AE, Enschede. Netherlands \\ ${ }^{2}$ Holst center, TNO, Eindhoven, Netherlands \\ ae.s.perdahcioglu@utwente.nl, bbo.hou@tno.nl, ca.h.vandenboogaard@utwente.nl
}

Keywords: instability, FLC, forming limit, stretch bending, sheet metal

\begin{abstract}
This research focuses on the prediction of the forming limit of certain Advanced High Strength Steel grades under stretch-bending conditions. For these types of steels it is experimentally observed and shown that when there is a bending component added to the main membrane deformation the formability predicted by the regular FLCs underestimate the material behavior. Due to the added effects of thickness stress due to contact and small radius bending as well as bending stresses, a through-thickness stress gradient forms which gives additional stability to the material beyond the forming limits determined by tests that generate mostly uniform membrane deformation. It is observed experimentally and by the detailed simulations that the cross-sectional stability is not lost instantaneously but gradually. A surface dent forms first on the outer surface and progresses in a stable manner towards the contact side since on the contact side the material has still potential to harden. This process delays the localization of the strains and stabilizes the formation of the local neck. For the prediction of this phenomenon theoretically and using shell elements, a modified incremental form of the maximum tension stability criterion is proposed to be applied at integration points through thickness. It is shown that with this criterion the phenomenon of gradual loss of stability can be captured during stretch-bending with shell elements.
\end{abstract}

\section{Introduction}

During sheet metal forming the material usually undergoes arbitrary deformation stages that include a combination of stretching and bending. When Advanced High Strength Steels (AHSS), such as CP (Complex Phase) or DP (Dual Phase) grades, undergo stretch-bending it is observed that the formability of the material is remarkably enhanced [1,2]. The formability of the material is usually considered as a material property which is determined experimentally and described using FLCs (Forming Limit Curves). The FLC of a material is used to predict the onset of formation of a local neck during forming. However the observation that addition of bending deformation influences formability suggests that a structural mechanism is also playing role in the stabilization of formation of the local neck.

Emmens and van den Boogaard [3] gave an overview of stabilizing mechanisms which lead to formability enhancement: 1) stabilization of necking due to avoided or postponed or reduced growth rate of necking; 2) shear stress and thickness stress will lower the yield stress in tension as a stabilizing mechanism; 3) major stress gradient through thickness induced by bending under tension (BUT) will lower the tension as a stabilizing mechanism. To include shear or thickness stresses, the traditional instability theories have been extended to non-planar stress states, by Eyckens et al. [4], Allwood et al. [5], Banabic et al. [6], Assempour et al. [7] and van den Boogaard et al. [8]. Their calculations predict enhanced forming limits due to the stabilizing effect of out-of-plane shear and thickness stresses. Xia et al. [9] and He et al. [10] studied the effect of BUT by employing Gaussian integration points across sheet thickness in traditional instability models. Vallellano et al. [11] suggested that necking under stretch-bending is mainly controlled by the development of damage in a partial volume located at the bottom of sheet.

In the current study the physical mechanism of enhanced formability are investigated using Finite Element (FE) simulations and theoretical considerations. Based on the findings an 
incremental stability indicator is proposed which can be implemented as either a post-processing tool or directly in a user defined material model.

\section{Physical mechanism of enhanced formability under stretch-bending}

During stretch-bending of sheet metal two main effects on the stress distribution can be observed. The first effect is due to bending where a through-thickness major stress gradient occurs, i.e. the major stress at the outer side is larger than that on the inner side. The second effect is related to the contact with the die, which is usually the way bending takes place during sheet metal forming and results in a thickness stress gradient, i.e. on the contact side there is a negative thickness stress and on the free side the thickness stress vanishes. These effects will be further elaborated in the following.

The presence of thickness stress $\left(\sigma_{33}\right)$ has two consequences on the material behavior. First of all, it reduces the in-plane major stress since the yield locus shifts in the direction of the hydrostatic stress, which is demonstrated in Fig. 1 by making use of Hill' 48 yield function. This means that the major stress at the contact side, which is already lower due to bending, will be reduced even more since the flow stress of the material will be lower.

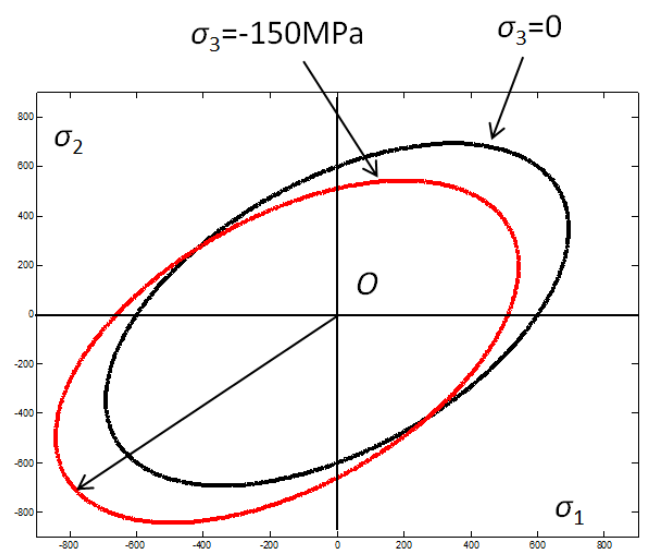

Figure 1: Hill' 48 yield surface in 2D principle stress space.

It can be concluded now that during bending there will be a major stress gradient through the thickness of the sheet and it will be increased when there is also a thickness stress gradient. Considering the fact that traditional shell element implementations are based on the plane stress assumption the extent of the increase of major stress gradient can be demonstrated by simulating an Angular Stretch Bend Test (ASBT) with solid elements and shell elements as shown in Fig. 2a. In Fig. $2 b$ it can be seen that with a decrease in punch radius the contact stresses increase significantly which reflects itself as an increase in the major stress gradient compute using solid elements. It is also worth mentioning that the effect is material dependent which relates to the fact that enhanced formability is mostly observed in AHSS.

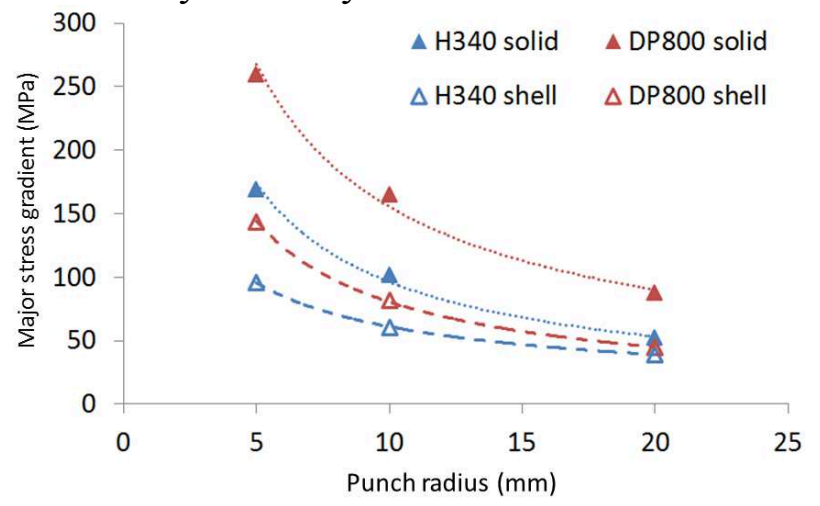

(a)

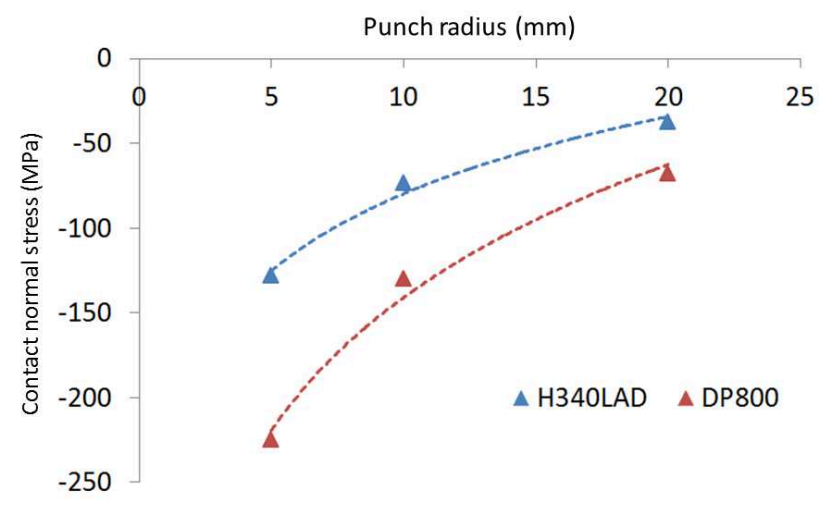

(b)

Figure 2: Uniaxial ASBT simulation results for DP800 and H340LAD: (a) Major stress gradient vs. punch radius, (b) Contact normal stress vs. punch radius. 
The other effect due to thickness stress can also be seen in Fig. 1 by comparing the location of the plane strain point on the two yield loci. When thickness stress is present the stress ratio at the plane strain point is lower than the normal one. This causes the strain ratio to be different (see Eq. 1) and in turn results in a different strain path during forming. Based on the formulation of Hill' 48 this can be calculated as:

$$
\rho=\frac{\mathrm{d} \varepsilon_{2}}{\mathrm{~d} \varepsilon_{1}}=\frac{F(\alpha-\beta)+H(\alpha-1)}{H(1-\alpha)+G(1-\beta)}, \quad \alpha=\frac{\sigma_{2}}{\sigma_{1}}, \quad \beta=\frac{\sigma_{3}}{\sigma_{1}}
$$

where $\rho$ is the incremental strain ratio, $\alpha$ and $\beta$ are the stress ratios and $F, G, H$ are parameters of the Hill' 48 yield function. This implies that the strain path during forming will be affected by the punch radius (see Fig. 2b) which is shown by solid element simulations of ASBT tests in Fig. 3.

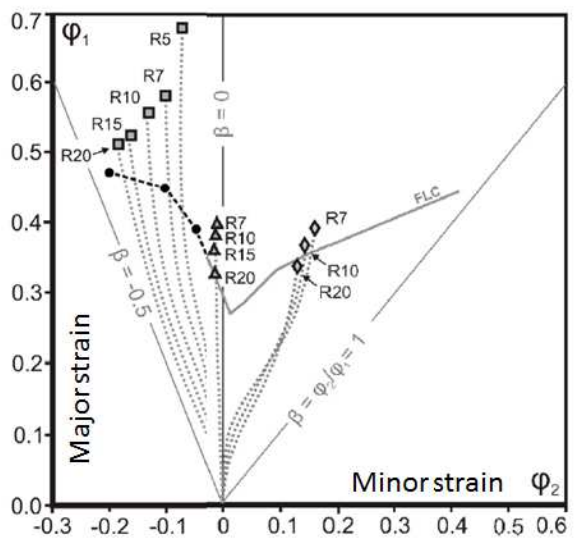

(a)

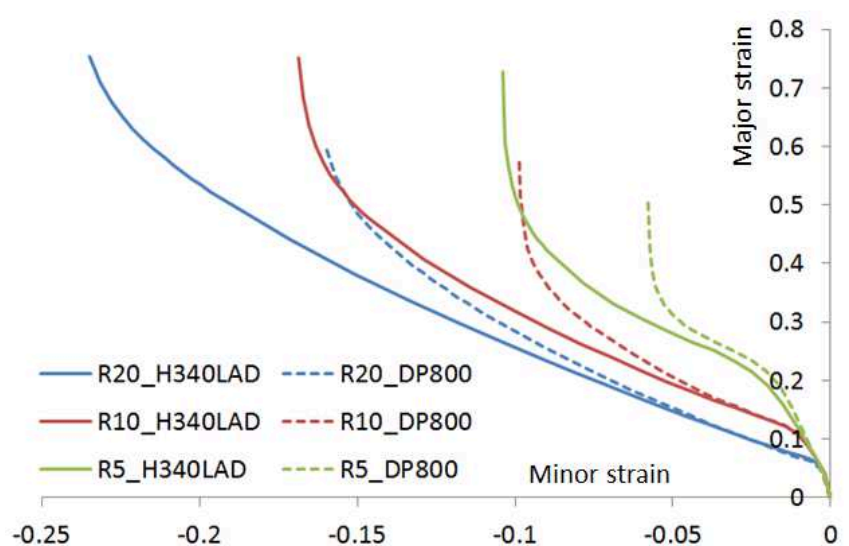

(b)

Figure 3: Non-linear strain path results from (a) ASBT experiment [10] and (b) ASBT simulation. In Fig. 3 it can be seen that as the punch radius decreases the change to plane strain state takes place earlier which means that actually formability is influenced by bending in a negative way.

Therefore it is clear that there are two competing factors in determining the formability of the material during stretch-bending, the enhancement due to major stress gradient and decrease due to strain path changes. As the experimental results demonstrate however usually the enhancement is the stronger of the two factors. In order to relate the major stress gradient to formability the major tension through the thickness of the sheet is plotted in Fig. 4 during an ASBT simulation. As expected there is a difference in the tension development between the top and the bottom halves of the sheet thickness. Moreover the maximum tension point also differs among the two. This suggests that the stability of the material is not uniform through the thickness and while the top half reaches maximum tension the bottom half still has capacity to harden.

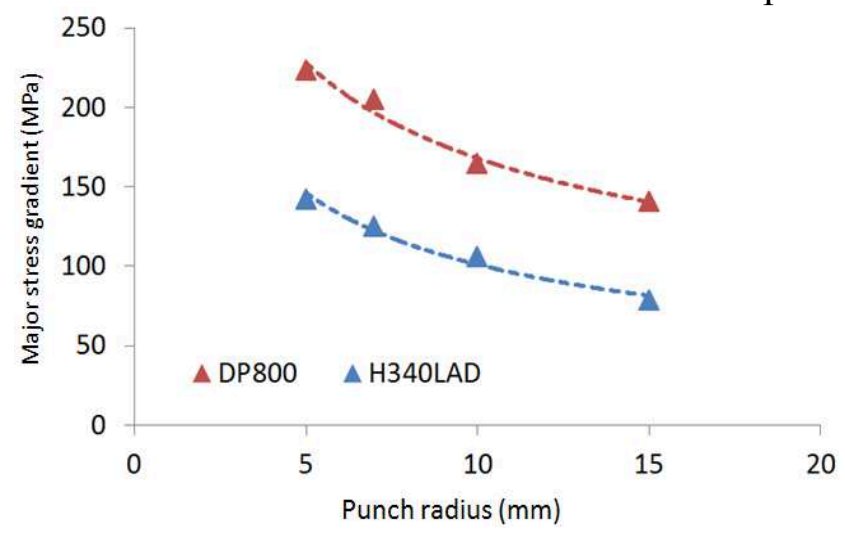

(a)

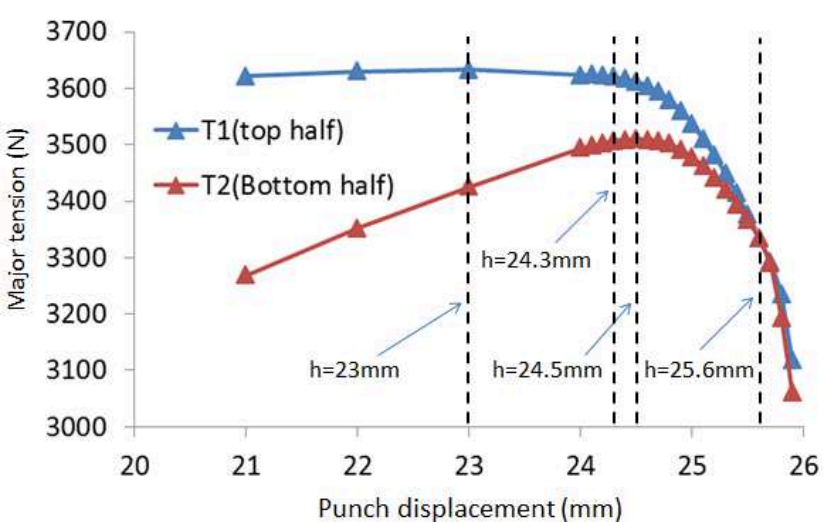

(b)

Figure 4: Plane-strain ASBT simulation results of DP800: (a) Major stress gradient vs. punch radius, (b) Major tension evolution vs. punch displacement $(h)$ with $R=10 \mathrm{~mm}$ 
As a result of this non-uniform stability distribution neck formation during stretch-bending is a gradual process and not an instantaneous one. This is shown in Fig. 5 where the cross sections of the sheet at different times during ASBT are plotted. It can be observed that a neck starts forming on the outer side and proceeds towards the inner side. While this happens however cross-sectional stability is not lost.

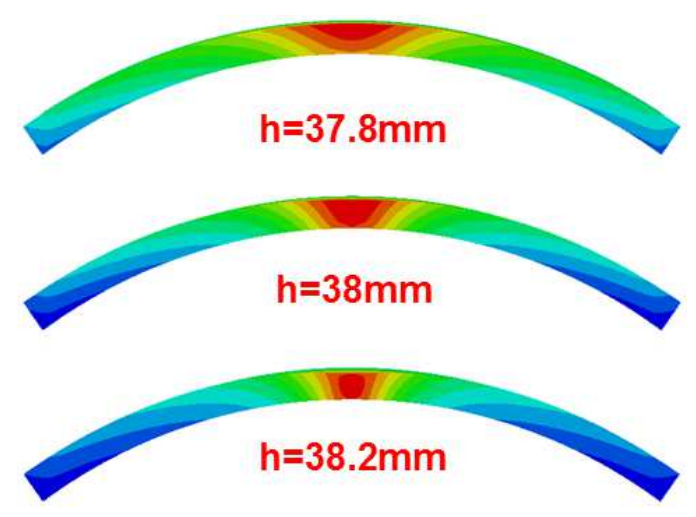

Figure 5: The maximum principal strain contour (thickness profile) from ASBT simulation at the onset of necking.

\section{Instability criterion}

According to Hill's maximum tension criterion [12] deformation is stable when the tension over the whole cross section of the sheet reaches a maximum value:

$$
\mathrm{d} T=\mathrm{d}\left(\sigma_{1} t\right)>0
$$

where $T$ denotes tension over the complete cross section and $t$ is the thickness. In this form the criterion applies to the complete cross section and monotonic loading paths since the differential is with respect to pseudo-time. In order to make this criterion applicable for non-proportional load paths and independent of thickness the following form is proposed:

$$
\frac{1}{T} \frac{\mathrm{d} T}{\mathrm{~d} \varepsilon_{1}^{*}}=\frac{1}{\sigma_{1}} \frac{\partial \sigma_{1}}{\partial \varepsilon_{1}^{*}}+\frac{\partial \varepsilon_{33}}{\partial \varepsilon_{1}^{*}}>0
$$

where $\varepsilon_{1}^{*}$ is the strain component in the direction of the major stress. This form is incremental, does not depend on the thickness and takes into account the current loading direction when considering the change in maximum tension. During unloading for instance while the form in Eq. 2 will predict instability the proposed equation will remain stable.

In a FE simulation the criterion is evaluated at the integration point level and reflects the stability of the volume spanned by the material (integration) point for the calculated strain increment. When the major tension increases for a strain increment in the same direction the material point is considered stable. In the case of shell elements it becomes convenient to track the stability of the cross section by considering the number of integration points that become unstable.

As discussed before in stretch-bending the plane stress assumption yields unreliable results. Therefore for reliable stress as well as instability predictions, a shell element that can simulate thickness stresses must be used. The results in this section are obtained with LS-Dyna by a shell element with thickness stress [13]. In Fig. 6 a uniaxial ASBT test simulation is shown. The prediction of instability is based on Eq. 3 and the output is a contour plot showing the number of integration points that are unstable at a given moment, i.e. green and red colors denote 1 and 7 (total) integration points, respectively. By looking at the contour plot therefore the extent of the stable neck can be tracked (see Fig. 5). 


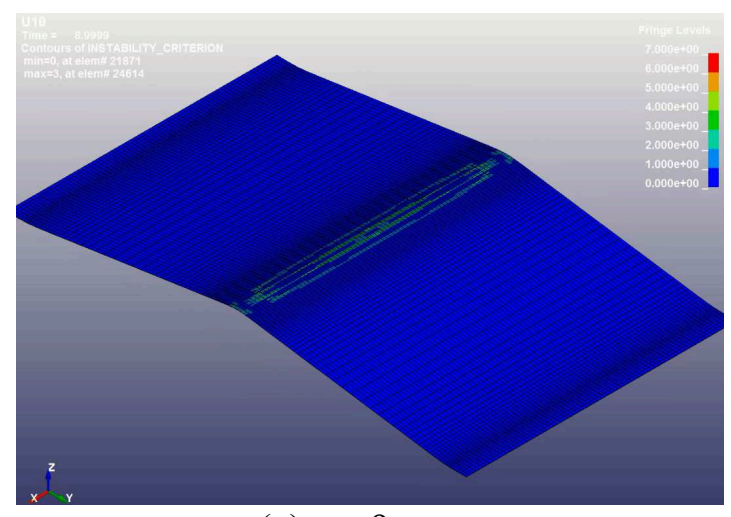

(a) $t=9 s$

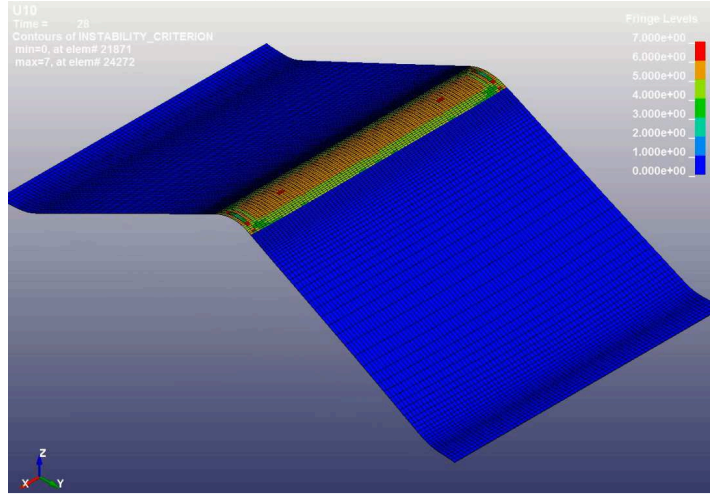

(c) $\mathrm{t}=28 \mathrm{~s}$

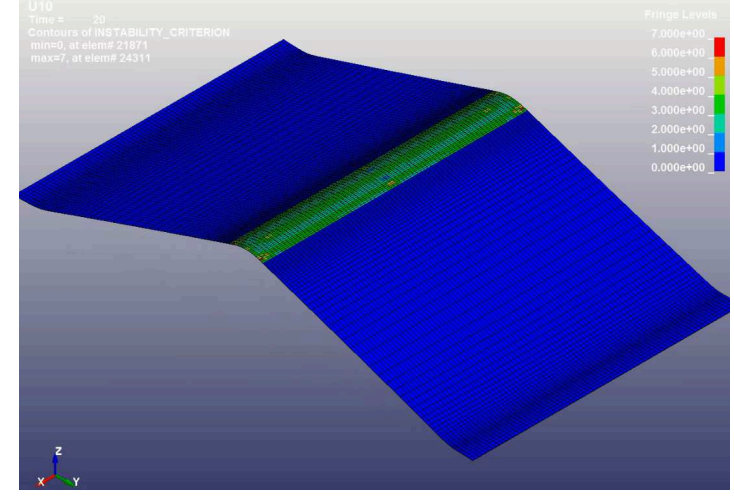

(b) $t=20 s$

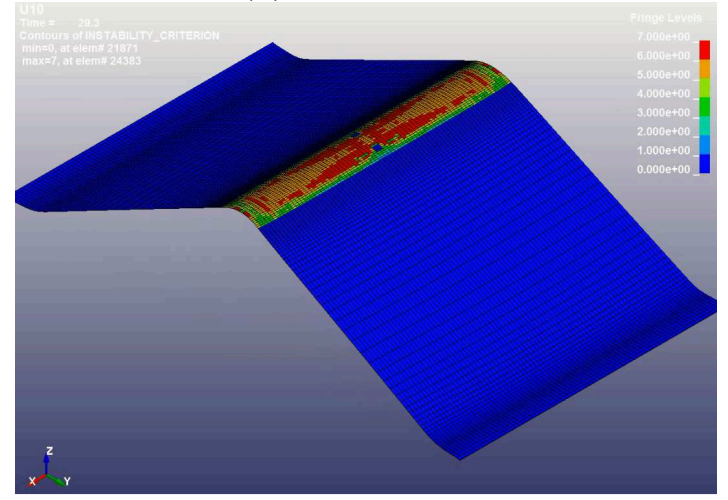

(d) $\mathrm{t}=29.3 \mathrm{~s}$

Figure 6: Instability evolution in uniaxial ASBT simulation of CP800 with $\mathrm{R}=10 \mathrm{~mm}$. Contours denote the number of integration points that have become unstable i.e. extent of necking.

Due to the fact that the criterion is stress-based it requires accurate prediction of stresses at each increment. This makes it less stable compared to strain-based criteria and may result in noisy behaviour in both space and time. Furthermore, numerically care must be taken to avoid numerical errors in case the strain and stress increments become small, such as at interface between localized zone and outside.

\section{Conclusion}

Using detailed FE simulations with solid elements the physical reasons behind the enhanced formability of AHSS under stretch-bending are clarified. The main reason is found to be the through-thickness major stress gradient because of which the stability behavior of the sheet is not uniform over the thickness. Although the outer side loses stability and a neck starts forming it is stabilized by the fact that the lower side can still carry load and does not localize. This results in a gradual loss of stability. The contributing factors to the major stress gradient are bending and thickness stress due to contact which are functions of the punch radius.

An opposing effect to formability enhancement is early strain path change towards plane strain with decreasing punch radius. Although this lowers the formability it is observed that the enhancement is greater and hence the overall result is increased formability.

A stability indicator is proposed and implemented for shell elements. It is based on the maximum tension criterion but computed in an incremental form. Modifications are made in order to be applicable in non-proportional loading and to eliminate thickness from the formulation.

\section{Acknowledgement}

The research leading to these results has received funding from the European Community's Research Fund for Coal and Steel (RFCS) under grant agreement n RFSR-CT-2011-00020. 


\section{References}

[1] E.T. Till, E. Berger, P. Larour, On an exceptional forming behaviour aspect of AHSS sheets, IDDRG 2008 International Conference. (2008) 429-440.

[2] E.T. Till, E. Berger, H. Pauli, D. Kitting, C. Walch, Towards accurate assessment of sheet forming processes with AHSS grades, IDDRG 2011 International Conference. (2011)

[3] W.C. Emmens, A.H. van den Boogaard, An overview of stabilizing deformation mechanisms in incremental sheet forming, J. Mater. Process. Tech. 209 (2009) 3688-3695.

[4] P. Eyckens, A. Bael, P. Houtte, An extended Marciniak-Kuczynski model for anisotropic sheet subjected to monotonic strain paths with through-thickness shear, Int. J. Plasticity. 27 (2011) 15771597.

[5] J.M. Allwood, D.R. Shouler, Generalised forming limit diagrams showing increased forming limits with non-planar stress states, Int. J. Plasticity. 25 (2009) 1207-1230.

[6] D. Banabic, S. Soare, On the effect of the normal pressure upon the forming limit strains, Numisheet 2008 International Conference. (2008) 199-204.

[7] A. Assempour, H.K. Nejadkhaki, R. Hashemi, Forming limit diagrams with the existence of through-thickness normal stress, Comput. Mater. Sci. 48 (2008) 504-508.

[8] A.H. van den Boogaard, W.C. Emmens, J. Huétink, Effect of thickness stress in stretchbending, NumiForm 2010 International Conference. (2010) 504-511.

[9] Z.C. Xia, D. Zeng, Sheet metal forming limit under stretch-bending, MSEC 2008 International Conference. MSEC_ICM\&P2008-72555.

[10] Ji He, Z.C. Xia, X.H. Zhu, D. Zeng, S.H. Li, Sheet metal forming limits under stretch-bending with anisotropic hardening, Int. J. Mech. Sci. 75 (2013) 244-256.

[11] D. Morales-Palma, C. Vallellano, F.J. García-Lomas, Assessment of the effect of the throughthickness strain / stress gradient on the formability of stretch-bend metal sheets, Materials \& Design. 50 (2013) 798-809.

[12] R. Hill, On discontinuous plastic states, with special reference to localized necking in thin sheets, J. Mech. Phys. Solids 1 (1952) 19-30.

[13] A. Haufe, K. Schweizerhof, P. DuBois, Properties \& Limits: Review of Shell Element Formulations, LS-Dyna Developer Forum. (2013) 